Running head: SPECIFICITY TRAINING

\title{
A meta-analysis and systematic review of Memory Specificity Training (MeST) in the treatment of emotional disorders.
}

Tom J. Barry ${ }^{1,2 *}$, Wing Yan Sze ${ }^{1}$, Filip Raes ${ }^{3}$

${ }^{1}$ Experimental Psychopathology Lab, Department of Psychology, The University of Hong Kong, Hong Kong.

${ }^{2}$ Researching Emotional Disorders and Development Lab, The Institute of Psychiatry, Psychology \& Neuroscience, King’s College London, London, United Kingdom.

${ }^{3}$ Centre for Learning Psychology and Experimental Psychopathology, University of Leuven, Leuven, Belgium

*Corresponding Author: Tom J. Barry

A: Department of Psychology, The University of Hong Kong, Jockey Club Tower, Pokfulam Road, Hong Kong

E: tom.j.barry@icloud.com; tjbarry@hku.hk

Abstract word count: 212

Word count: 9421

Figures: 6

Table: 1

Disclosures: This manuscript was not supported by any external funding. The authors have no conflicts of interest to acknowledge. 


\begin{abstract}
The tendency to recall events from one's past in a non-specific and overgeneral way has been found to predict the onset and severity of a range of emotional disorders. Memory Specificity Training (MeST) was devised a decade ago in order to target and modify this tendency so as to reduce the symptoms of emotional disorder or to reduce the risk that such disorders might emerge over time. We present a meta-analytical review of research into the effects of MeST on autobiographical memory specificity in the context of emotional disorders $(k=12)$. MeST was associated with substantial improvement in memory specificity $(d=-1.21)$ and depressive symptoms $(d=0.47)$ and MeST groups outperformed control groups at postintervention in terms of specificity $(d=1.08)$ and depressive symptoms $(d=-.29)$. However, these effects were transitory and the benefit of MeST over control groups was mostly lost by follow-up assessment. There was mixed evidence in terms of MeST's effects on other processes associated with reduced specificity but MeST showed most promise in improving problem solving abilities and hopelessness. MeST holds promise as a novel intervention targeting reduced specificity but future studies are warranted with control groups that enable the investigation of MeST's mechanism of action and in studies with larger and more varied samples.
\end{abstract}

Keywords: Depression; Posttraumatic Stress Disorder; Aging; Schizophrenia; Treatment; Memory 
Cognitive-behavioural therapy (CBT) for emotional disorders such as Major Depressive Disorder (MDD), is effective and yet it is imperfect (Cuijpers, Cristea, Karyotaki, Reijnders, \& Huibers, 2016; Linde et al., 2015). For example, CBT shows very large effect sizes in the treatment of MDD when considering evidence from randomised controlled trials $(g=.75$; Cuijpers et al., 2016). However, when one takes into account the presence of publication bias in this literature, as well as the use of inadequate, passive controls (e.g., wait-list), and other aspects of poor research quality (e.g., poor blinding, randomization and management of incomplete data), effect sizes become small to moderate in the treatment of depression $(g=$ .43) and other emotional disorders (Cuijpers et al., 2016). Furthermore, the effects of CBT in treating depression are comparable to other common talking therapies (Linde et al., 2015). Some authors have concluded that greater focus must now be given to developing interventions that target specific mechanisms associated with disorder maintenance, such as those related to cognitive impairments in memory or attention, rather than the non-targeted, fix-all approach of CBT (Craske, 2018). Such interventions might also offer promise in the prevention of emotional disorders as many cognitive impairments are also associated with disorder vulnerability and yet little is currently done to target these mechanisms and prevent the emergence of emotional disorders (van Zoonen et al., 2014). There is also some suggestion that universal, non-targeted prevention programmes may be less effective than approaches which target particular disorder mechanisms (Werner-Seidler, Perry, Calear, Newby, \& Christensen, 2017). One such targeted intervention that has been used to both prevent and treat emotional disorder is Memory Specificity Training (MeST; Raes, Williams, \& Hermans, 2009).

MeST offers promise in both treatment and prevention given the breadth of research suggesting that the reduced autobiographical memory specificity (rAMS) which it targets, is evident amongst people with depression and other clinical diagnoses (Van Vreeswijk \& De 
Wilde, 2004) and can predict increases in symptom severity across time (Kleim \& Ehlers, 2008; Sumner, Griffith, \& Mineka, 2010). By improving memory specificity problems through intervention, we would therefore expect a commensurate improvement in the symptoms of emotional disorder in currently symptomatic people, or a reduction in the likelihood of acquiring a diagnosis in those people not currently symptomatic. However, one must ask how MeST has been delivered, how it has been investigated and how effective it has been in improving symptoms and other outcomes. From this one must ask whether MeST or its investigation can be improved and what form such improvements might take. The present meta-analysis and systematic review sought to answer these questions by collating and reviewing all existing evidence regarding MeST and other interventions within which components of MeST can be found.

It is of note that there has been a recent review of the broader literature of memory training protocols (Hitchcock, Werner-Seidler, Blackwell, \& Dalgleish, 2017). However, this review excluded studies that did not include control comparisons and which involved healthy participants or participants with diagnoses other than depressive and anxious disorders. As such, they included three interventions that trained memory specificity (Moradi et al., 2014; Neshat-Doost et al., 2013; Serrano, Latorre, Gatz, \& Montanes, 2004) and did not evaluate the full breadth of available MeST literature.

\section{Reduced autobiographical memory specificity and its causes and consequences}

In order to fully evaluate MeST we must first consider what rAMS is, what causes it and how MeST attempts to improve it. rAMS - or overgeneral memory - refers to the difficulty some people have in recalling specific experiences from their past. Individual differences in memory specificity are typically assessed using the Autobiographical Memory Test (AMT) where participants are given several cue words of positive, negative or neutral valence and they are asked to recall a memory related to this cue (Williams \& Broadbent, 1986). 
Although there is debate regarding the way in which AMT responses should then be coded, the overwhelming psychometric consensus is that individual differences in specificity are best operationalised as the number, or proportion of memories recalled within the AMT that are specific, or those which involve a single event lasting 24 hours or less (Griffith et al., 2009; Griffith et al., 2012; Heron et al., 2012; Takano, Gutenbrunner, Martens, Salmon, \& Raes, 2017). MeST therefore seeks to increase the number or proportion of specific memories recalled from pre- to post- intervention.

It is also important to consider processes which are thought to reduce memory specificity, as well as those which are thought to be a consequence of rAMS and which mediate its association with emotional disorders as many of these processes are measured within MeST investigations. We might then evaluate the extent to which MeST influences these processes. In accordance with Conway and Pleydell-Pierce's (2000) model of memory retrieval, Williams (2006) and Williams et al. (2007) suggested that memory retrieval follows a hierarchical process where abstract, categorical themes are first retrieved and then specific, concrete events are retrieved. rAMS is therefore characterised by retrieval that fails to work its way past the abstract level. Williams (2006) CaRFAX model suggested three pathways through which this can occur, each of which is thought to be a prominent feature of emotional disorders: Capture and Rumination, Functional Avoidance and impaired eXecutive functioning. During retrieval of a memory related to a cue, a depressed person might be Captured by, and then Ruminate on, semantically-associated, self-related, negative conceptual themes (e.g., attempts to retrieve a memory of a fun time may evoke thoughts of being boring) or categories of events (e.g., when one's grandchildren used to visit them). This ruminative thinking is repetitive, self-concerned and unconstructive. Also, one might try to avoid specific events in one's memories, for fear that such memories might evoke negative feelings - so called Functional Avoidance (e.g., one might avoid recalling memories of 
specific social gatherings for fear that one might retrieve a memory of a negative encounter that occurred). The final pathway, impaired eXecutive functioning, is suggested to compromise retrieval independently of the other pathways. A person with weak executive functioning may be less able than a person with strong functioning to hold a specific memory in mind during retrieval or to update this memory as other memories are retrieved (Sumner, 2012). They may also be less able to inhibit distraction by irrelevant thoughts or other ongoing events when they are trying to retrieve a memory or when they are trying to verbalise it (Sumner, 2012).

Through these three pathways, and the interaction between or combination of pathways, memory specificity comes to be comprised, with several consequences that may then in turn lead to emotional disorder. First, reduced specificity may compromise one's abilities to solve problems as one is less able to use information from previous events to inform the selection of solutions for ongoing problems (Arie, Apter, Orbach, Yefet, \& Zalzman, 2008). This is also thought to compromise one's ability to imagine and plan for one's personal future, leading to feelings of hopelessness and excessively negative views regarding one's future (Arie et al., 2008; Evans, Williams, O’loughlin, \& Howells, 1992). Relatedly, rAMS may also encourage one to think in a more verbal, ruminative manner (Raes, Williams, \& Hermans, 2008) and prevent one from thinking in a more constructive imagery-based manner (Holmes, Blackwell, Burnett Heyes, Renner, \& Raes, 2016). Also, by not recalling specific negative events that have happened in our past, this is thought to reduce exposure to negative emotional experiences and so restricting one's ability to learn how to regulate and cope with these emotions when they are inevitably evoked (Harris et al., 2015; Hermans, Raes, \& Eelen, 2005).

MeST research has therefore not only examined the effects of MeST on memory specificity and the symptoms of emotional disorders, but also its effects on the processes 
associated with reduced specificity. Our review therefore considers evidence regarding these other processes where it exists.

The present meta-analysis and systematic review provides the first synthesis of all existing studies in which MeST has been tested within the context of emotional disorders. Following the initial search for and extraction of data, the review begins with a detailed introduction to MeST and the ways in which it has been translated and implemented. The effects of MeST on individual differences in memory specificity, the symptoms of emotional disorders and other associated processes are then analysed. Our discussion then synthesises these findings and considers the current state of MeST and future directions.

\section{Method}

\section{Review strategy, inclusion criteria and data extraction}

All studies were sampled from the authors' personal libraries $(k=12$; one study reported two relevant intervention groups so final $k=13$ ). Studies were included in the review if they involved a training protocol for enhancing the specificity of autobiographical memory amongst participants and where there was an assessment of change in the symptoms of emotional disorders. Studies were not included in this review if they involved memory training amongst participants characterised by cognitive impairments (Emsaki, NeshatDoost, Tavakoli, \& Barekatain, 2017). To ensure that no studies were missed a search of the PsycINFO, PsycARTICLES and MEDLINE databases was undertaken, using the terms 'memory specific* training' and 'specific* training'. This search, performed in January 2018 by the second author, returned 38 articles. A search of the titles and abstracts of these articles did not reveal any new articles which met the inclusion criteria, beyond those already identified. 
Several of the studies identified did not explicitly refer to MeST but the training protocols that they tested were highly similar to the MeST protocols used elsewhere (Blairy et al., 2008; Celano et al., 2016; Leahy, Ridout, Mushtaq, \& Holland, 2017; Ricarte, Hernández-Viadel, Latorre, \& Ros, 2012; Serrano et al., 2004). We include these studies in our review and the differences between these protocols and the other MeST studies is considered below. For simplicity, we refer to each of these variations simply as MeST except in one study that included both MeST and Life Review groups (e.g., Leahy et al., 2017). See Table 1 for an outline of MeST studies and their sample characteristics.

Where available, the first author extracted a) participant characteristics (sample size, mean age, proportion of females, primary diagnosis); mean and standard deviations at preand post- intervention and follow-up assessments for b) memory specificity scores c) the symptoms of emotional disorders and, d) other process outcomes (e.g., rumination, executive functioning, problem solving etc.); e) intervention characteristics (number and duration of sessions, whether it was conducted in an individual or group format, and if it was delivered in-person or online); and finally, f) study characteristics (information on control conditions, follow-up length, drop-out rates). Where mean and standard deviations were not available, the authors were contacted and data were requested. All missing data was acquired and as such no studies were excluded from our analyses. A research assistant extracted data from three of the 13 studies included in the analyses ( $25 \%$ of all studies) to ensure that the first author had extracted the data correctly. The intra-class coefficient was .997 , with a single discrepancy related to the way a follow-up period was recorded for one study; all other extracted data were equivalent between raters.

Our literature search identified several studies where specificity was induced in a more temporary, experimental setting. These studies (e.g., Jing, Madore, \& Schacter, 2016; Madore \& Schacter, 2014) did not aim to improve rAMS that is found within emotional 
disorders, and as such they are not included within our main review of MeST. However, these studies explored the effects of their specificity induction on processes similar to those that have been associated with rAMS. These studies are presented within our discussion.

\section{Data preparation and analysis}

The meta-analysis was conducted using the R (3.5.1)(R Development Core Team, 2011) metaphor (2.0-0) package (Viechtbauer, 2010). Standardised mean differences (Cohen's d) were computed for change in each dependent variable (specificity, emotional disorder symptoms and other process outcomes such as problem-solving abilities) from pre- to postintervention and post-intervention to follow-up within the MeST group. These analyses examined the extent to which these variables improved as a result of MeST and, in the case of the post- to follow-up analysis whether there was evidence of continued improvement or a decline following intervention completion. Effect sizes were weighted based on the sample size within each study. Standardised mean differences were also computed for between-group differences at post-intervention and follow-up. Where there were two or more studies that examined the same dependent variables at the same time points, these mean difference scores were pooled and meta-analysed using a random-effects framework (Field \& Gillett, 2016). The extent to which pooled effect sizes differed from zero was assessed using a $Z$ test. The extent to which there was heterogeneity in study effect sizes was assessed using a $Q$ test and the amount of heterogeneity between studies was represented using $I^{2}$ and $\tau^{2}$ statistics. Where there was evidence of significant heterogeneity and where there was a sufficient number of effect sizes to warrant further analyses we followed this up with sub-group analyses (e.g., comparisons of active and passive controlled studies).

As most studies examined specificity and depressive symptoms we were able to examine the potential for publication bias within these analyses. Given the limited number of studies for other dependent variables this was not possible. For specificity and depressive 
symptoms we first visually inspected funnel plots and then used Egger's test to assess whether the effect size of each study was related to its sample size. All funnel plots are available in the online supplement.

\section{Results}

\section{What is Memory Specificity Training?}

Serrano et al. (2004) presented the first implementation of a protocol for training memory specificity. However, their protocol was included within a wider programme of Life Review Therapy. The first implementation of a dedicated protocol for Memory Specificity Training was conducted by Raes, Williams and Hermans (2009) in their pilot investigation of MeST amongst depressed inpatients. This study comprised of four, one-hour group sessions. Although other MeST investigations differ in terms of the number and length of sessions, the basic format and content of MeST is for the most part equivalent between studies.

Typically, in the first session participants are given introductory psychoeducational information on memory functioning within depression (e.g., mood-congruent/dependent memory retrieval) and in particular the concept of autobiographical memory specificity. Participants are also asked to recall, and discuss within the group, specific memories for positive and neutral cue words and they are told to try to recall as much spatio-temporal and contextual detail as possible. The therapist gives feedback on the specificity of these memories and guides the participants in how specificity might be improved. In the second session, participants are asked to recall two unique memories for each of several positive and neutral cue words. This practice is intended to reduce overgeneralisation between memories that are related to the same cue word by focusing on the aspects of memories that make them unique. Session three replicates the practice of session two but where the previous sessions used positive and neutral cue words, session three also involves negative cue words. Again, the uniqueness of memories is emphasised in order to teach participants not to generalise 
memories from positive cue words (e.g., memories of skill) to memories from negative cue words (e.g., memories of clumsiness). Session four then focuses on encouraging metacognitive awareness and teaching participants about the conditions in which one might shift from specific retrieval to more general retrieval, such as during stressful situations. Participants are given homework between each session where they are asked to recall and note down several specific memories on a nightly basis. The four sessions can be summarised as psychoeducation, uniqueness training, introducing negative memories and metacognitive awareness. Other investigations of MeST have increased the number of sessions in order to more gradually introduce cues of different valences between sessions, such as by starting with positive, then negative and then neutral as in the trial by Neshat-Doost et al. (2013). Other studies which involve more sessions have included greater practice and review of homework but with no additional therapeutic components ( 5 sessions: Eigenhuis, Seldenrijk, van Schaik, Raes, \& van Oppen, 2017; 6 sessions: Maxwell et al., 2016; 7 sessions: Takano, Moriya, \& Raes, 2017).

Several other studies have tested variations on MeST with additional therapeutic components or with different delivery formats. Regarding the latter, MeST has been translated for online or computerised delivery without therapist interaction. In computerised MeST (c-MeST; Takano, Moriya, et al., 2017) a machine learning algorithm, that has elsewhere been used to code autobiographical memories according to their specificity (Takano, Ueno, et al., 2017; Takano, Gutenbrunner, Martens, Salmon, \& Raes, 2018), codes the memories given during MeST sessions and then offers feedback to participants regarding how they might improve their specificity. c-MeST involves seven once-a-day sessions of variable length (15-45 minutes) that focus on cued retrieval, without the modules of MeST which involve psychoeducation and metacognitive awareness. A variation of MeST has also been tested in a workbook format but where the modules of the workbook are introduced and 
reviewed by a clinician. This Cognition Focused workbook (Celano et al., 2016) trains specificity by asking participants to recall three neutral events from each day over the past week in as much detail as possible whilst avoiding assigning emotions to these events. In the first session, the details of these memories are then reviewed by a clinician in-person. The participant then reviews the workbook at home. Weekly recall exercises are given, where participants focus on events of different kinds and from different settings. These memories are then reviewed on a weekly basis via telephone (Celano et al., 2016).

Other interventions have trained memory specificity whilst also including therapy components related to other aspects of psychopathology. For example, in Blairy et al.'s (2008) Autobiographical Memory Intervention emphasis is placed on personal identity and self-defining adjectives and the development of goals that are in line with the way people define themselves. This study also incorporated aspects of game-ification whereby participants were given points based on the number of details they could recall for a given memory. The principles of MeST have also been combined with Life Review Therapy where additional emphasis is placed on how one's memories fit within the context of the stages of their life (childhood, adolescence, adulthood) and also focuses solely on positive memories rather than on negative or neutral memories as in traditional MeST (Leahy et al., 2017;

Ricarte et al., 2012; Serrano et al., 2004).

\section{The effects of MeST on Autobiographical Memory Specificity}

One study included in this review did not examine the effects of their intervention on memory specificity (Celano et al., 2016) and as such was excluded from this analysis, though it was included in other analyses.

Within-group change

$\operatorname{MeST}(k=12)$ was associated with large improvement from pre- to post- intervention $(d=$ $1.21,95 \% \mathrm{CI}[-1.60,-0.81], Z=-6.03, p<.001)$. There was also a substantial amount of 
heterogeneity between studies in the size of these effects $\left(\tau^{2}=.32, I^{2}=71.1 \%, Q(11)=31.55\right.$, $p<.001)$. Amongst tests of change in specificity from post-intervention to follow-up $(k=7)$, there was no evidence of continued improvement after MeST was complete $(d=0.12,95 \%$ $\mathrm{CI}[-0.14,0.37], Z=0.88, p=.381)$ and there was no evidence of heterogeneity between studies $\left(\tau^{2}=.00, I^{2}=3.61 \%, Q(6)=4.88, p=.559\right)($ See Figure 1$)$.

\section{Between-group differences}

At post-intervention $(k=10)$, relative to control groups, MeST groups showed significantly higher specificity scores $(d=1.08,95 \% \mathrm{CI}[0.45,1.71], Z=3.35, p<.001)$. There was also a substantial amount of heterogeneity between studies $\left(\tau^{2}=.87, I^{2}=86.8 \%, Q(9)=38.56, p<\right.$ $.001)$. Two additional analyses were performed to examine whether similar results were evident in studies with passive waitlist control groups $(k=4)$ and active control groups $(k=$ 6). MeST groups outperformed control comparisons in studies with waitlist control groups ( $d$ $=1.90,95 \% \operatorname{CI}[0.66,3.14], Z=3.00, p=.003)$ and active control groups $(d=0.68,95 \%$ $\mathrm{CI}[0.26,1.09], Z=3.21, p=.001)($ See Figure 2).

At follow-up, overall $(k=6)$, MeST outperformed control comparisons although this effect was not significant $(d=0.98,95 \% \mathrm{CI}[-0.12,2.07], Z=1.74, p=.081)$ and there was substantial variability between studies in the size of the group difference at follow-up $\left(\tau^{2}=\right.$ $\left.1.70, I^{2}=92.7 \%, Q(5)=34.89, p<.001\right)$. Again, in sub-group analyses for active $(k=4)$ and waitlist $(k=2)$ control studies MeST groups did not outperform active control comparisons at follow-up $(d=0.33,95 \% \mathrm{CI}[-0.16,0.83], Z=1.31, p=.191)$ but did outperform waitlist comparisons $(d=2.55,95 \% \mathrm{CI}[0.02,5.07], Z=1.98, p=.048)$ (See Figure 2$)$.

MeST was associated with substantial improvement in memory specificity and outperformed both active and passive control groups at post-intervention. However, MeST only continued to outperform waitlist controls groups by follow-up assessment and not active control groups. 


\section{Publication bias}

Visual inspection of funnel plots (see online supplement Figure s1) suggested that there was some evidence of publication bias in the analyses of specificity. This was confirmed by Egger's test on change in specificity from pre- to post $(Z=-3.85, p<.001)$ and there was some evidence of asymmetry for group differences at post-intervention $(Z=1.66, p$ $=0.096)$ and follow-up $(Z=1.91, p=0.056)$. There was no evidence of significant asymmetry in the post-intervention to follow-up analyses $(Z=0.58, p=0.559)$.

\section{The effects of MeST on depression symptoms}

\section{Within-group change}

Overall $(k=13)$, MeST was associated with improvement in depression symptoms from preto post- intervention $(d=0.47,95 \% \mathrm{CI}[0.22,0.72], Z=3.71, p<.001)$ and there was a significant amount of heterogeneity between studies in the size of these effects $\left(\tau^{2}=.09, I^{2}=\right.$ $46.2 \%, Q(12)=21.76, p=.040)$. Amongst tests of change in depression symptoms from post-intervention to follow-up $(k=9)$, there was no evidence of continued improvement after $\operatorname{MeST}(d=0.02,95 \% \mathrm{CI}[-0.20,0.23], Z=.15, p=.883)$ and there was no evidence of heterogeneity between studies $\left(\tau^{2}=.00, I^{2}=0 \%, Q(8)=10.39, p=.239\right)$ (See Figure 3). Between-group change At post-intervention $(k=11)$, relative to control groups, MeST groups showed significantly lower depression scores $(d=-0.29,95 \% \mathrm{CI}[-0.48,-0.10], Z=-2.93, p=.003)$ and there was no evidence of heterogeneity between studies $\left(\tau^{2}=.00, I^{2}=0 \%, Q(10)=10.08, p=.433\right)$. For illustrative purposes and given the differences between active and waitlist controls in terms of specificity, we repeated the analyses for each sub-group. MeST groups outperformed control comparisons in studies with waitlist control groups $(k=4, d=-0.57,95 \% \mathrm{CI}[-0.96,-0.18], Z$ $=-2.87, p=.004)$ but not studies with active control groups $(k=7, d=-0.17,95 \% \mathrm{CI}[-0.40$, $0.06], Z=-1.44, p=.151)$ (See Figure 2). 
At follow-up $(k=8)$, MeST groups did not outperform control comparisons $(d=-$ $0.17,95 \% \mathrm{CI}[-0.42,0.08], Z=-1.34, p=.180)$ and there was no evidence of heterogeneity between studies $\left(\tau^{2}=.02, I^{2}=12.2 \%, Q(7)=10.07, p=.185\right)$ (See Figure 4).

MeST was associated with substantial improvement in depressive symptoms and also outperformed control groups at post-intervention. However, MeST only outperformed waitlist controls and not active control groups. Any advantage of MeST compared to control groups was lost by follow-up assessment.

\section{Publication bias}

Visual inspection of funnel plots (see online supplement Figure s2) suggested that there was little evidence of publication bias in the analyses of depressive symptoms. Egger's test also suggested that there was no evidence of asymmetry in the pre- to post- $(Z=.14, p=$ $.889)$ or post- to follow-up analyses $(Z=-1.17, p=.242)$ or the group difference analyses at post-intervention $(Z=-.303, p=.762)$ or follow-up $(Z=-.18, p=.859)$.

\section{The effects of MeST on post-traumatic stress symptoms}

Only two studies examined the effects of MeST on post-traumatic stress symptoms (PTSS; Maxwell et al., 2016; Moradi et al., 2014), though both involved follow-up assessments and a control comparison.

\section{Within-group change}

Across these studies, there was no evidence of a significant change in PTSS from pre- to post- intervention for MeST groups $(d=2.09,95 \% \mathrm{CI}[-0.98,5.08], Z=1.37, p=.169)$ although there was substantial variability between the studies $\left(\tau^{2}=4.28, I^{2}=92.4 \%, Q(1)=\right.$ 13.16, $p<.001)$. Moradi et al. (2014) showed a considerable improvement in symptoms from pre- to post $(d=3.64,95 \% \mathrm{CI}[2.34,4.95])$ whereas Maxwell et al. (2016) showed small-tomoderate improvement $(d=0.60,95 \% \mathrm{CI}[-0.40,1.60])$.

Regarding change from post-intervention to follow-up, there was also no evidence of 
a significant change in PTSS $(d=0.08,95 \% \mathrm{CI}[-0.74,0.91], Z=0.20, p=.840)$ and there was no evidence of variability between the studies $\left(\tau^{2}=.15, I^{2}=40.7 \%, Q(1)=1.69, p=\right.$ $.194)$.

\section{Between-group differences}

Similarly, the pooled effect size for group differences at post-intervention suggested that MeST groups did not outperform control comparisons $(d=-0.98,95 \% \mathrm{CI}[-3.84,1.88], Z=-$ $0.67, p=.503)$, however, there was considerable variability between studies $\left(\tau^{2}=4.00, I^{2}=\right.$ 93.6\%, $Q(1)=15.57, p<.001)$. MeST outperformed a waitlist control (Moradi et al., 2014; $d$ $=-2.45,95 \% \mathrm{CI}[-3.50,-1.39])$ but not an active Cognitive Processing Therapy control (Maxwell et al., 2015; $d=0.48,95 \%$ CI[-0.52, 1.47]).

These findings were further replicated at follow-up assessment, where MeST did not outperform control comparisons in terms of the pooled effect sizes across studies $(d=-.99$, $95 \% \mathrm{CI}[-3.58,1.60], Z=-.75, p=.453)$. However, there was considerable variability between studies $\left(\tau^{2}=3.22, I^{2}=92.37 \%, Q(1)=13.10, p<.001\right)$ where, MeST again outperformed waitlist control (Moradi et al., 2014; $d=-2.31,95 \%$ CI[-3.35, -1.28]) but not Cognitive Processing Therapy (Maxwell et al., 2015; $d=0.32,95 \%$ CI[-0.66, 1.31]).

Across the two studies to assess PTS symptoms, there was mixed evidence of an improvement in these symptoms due to MeST across time or relative to control groups.

\section{The effects of MeST on CaRFAX mechanisms}

\section{Rumination}

Two studies (Raes et al., 2009; Werner-Seidler et al., 2018) reported change in rumination from pre- to post- intervention amongst participants who received MeST. The pooled effect size for change in rumination was small and did not differ significantly from zero $(d=0.26$, $95 \% \mathrm{CI}[-0.30,0.72], Z=1.14, p=.253)$ and there was no evidence of heterogeneity between individual study effect sizes $\left(\tau^{2}=0.0, I^{2}=0.0 \%, Q(1)=0.12, p=.730\right)$. 
Only Werner-Seidler et al. (2018) assessed rumination at follow-up and used a control group comparison. They found only small but non-significant change in rumination from post-intervention to follow-up $(d=0.32,95 \% \mathrm{CI}[-0.21,0.85])$ and only small differences between MeST participants and psychoeducational control participants at post-intervention $(d$ $=-.06,95 \% \mathrm{CI}[-0.59,0.47])$ and follow-up $(d=-.44,95 \% \mathrm{CI}[-0.99,0.12])$.

\section{Executive functioning}

Three studies assessed aspects of executive functioning, two of which compared MeST to a control group in terms of verbal fluency at pre- and post- intervention. Across these studies there was only trivial change in verbal fluency from pre- to post- MeST $(d=-.15,95 \% \mathrm{CI}[-$ $0.57,0.26], Z=-.71, p=.476)$ and there was no evidence of heterogeneity between the studies $\left(\tau^{2}=0.0, I^{2}=0.0 \%, Q(1)=.47, p=.492\right)$. Both Blairy et al. $(2008 ; d=0.05,95 \% \mathrm{CI}[-$ $0.660 .77])$ and Werner-Seidler et al. $(2018 ; d=-.25,95 \% \mathrm{CI}[-0.76,0.25])$ showed only small change in verbal fluency from pre- to post.

Only Werner-Seidler et al. (2018) examined change from post- to follow-up assessment and their analysis revealed a small change in verbal fluency across these assessments and the confidence intervals overlapped with zero $(d=-.14,95 \% \mathrm{CI}[-0.67$, $0.39])$.

Comparisons with control groups at post-treatment $(k=2)$ suggested that MeST outperformed control comparisons $(d=0.55,95 \% \mathrm{CI}[0.11,0.99], Z=2.43, p=.015)$ and there was no evidence of heterogeneity between the studies $\left(\tau^{2}=0.0, I^{2}=0.0 \%, Q(1)=.19, p\right.$ $=.662)$. MeST showed small-to-moderate improvement in verbal fluency versus psychoeducational control comparisons (Werner-Seidler et al.., 2018; $d=0.62,95 \%$ CI[0.08, 1.16]; Blairy et al., $d=0.41,95 \%$ CI[-0.36, 1.18]). For Werner-Seidler et al. (2018) this relative improvement became stronger by follow-up assessment $(d=1.07,95 \% \mathrm{CI}[0.49$, $1.66])$. 
Blairy et al. (2008) also examined change in digit forward and backward spans amongst MeST participants and their psychoeducational control group. MeST was associated with trivial improvement in digit forward $(d=-0.07,95 \% \mathrm{CI}[-0.78,0.65])$ and backward spans $(d=-0.15,95 \% \mathrm{CI}[-0.87,0.57])$ from pre- to post and confidence intervals for the group comparisons for both indices suggested that MeST participants did not consistently outperform controls for either index (digit forward: $d=0.58,95 \% \mathrm{CI}[-0.20,1.35]$; digit backward: $d=0.62,95 \% \mathrm{CI}[-0.16,1.39])$.

Similarly, in their comparison of OSPAN performance between MeST participants and waitlist control, Takano et al. (2017) found only small improvement from pre- to postintervention for MeST participants $(d=-.41,95 \% \mathrm{CI}[-1.02,0.20]$ and MeST participants did not consistently outperform waitlist control participants at post-intervention $(d=0.22,95 \%$ CI[-0.40, 0.84].

MeST was associated with improvement in verbal fluency relative to control interventions, but showed no improvement in other measures of executive functioning.

\section{The effects of MeST on process outcomes}

\section{Means-End Problem Solving}

Amongst comparisons of improvement in Means-End Problem Solving (MEPS) task performance from pre- to post- intervention $(k=4)$ there was evidence of only trivial change for MeST participants in both steps $(d=-.16,95 \% \mathrm{CI}[-0.46,0.15], Z=-1.00, p=.149)$ and effectiveness $(d=-.06,95 \%$ CI[-0.36, 0.25], $Z=-.38, p=.708)$ indices. Neither analysis showed any evidence of heterogeneity between studies $\left(\tau^{2}\right.$ and $I^{2}$ estimates both 0 , largest Q $=2.16$, smallest $\mathrm{p}=.539)($ See Figure 5).

In analyses of improvement from post-intervention to follow-up assessments $(k=2)$ there was also evidence of small-to-moderate, but non-significant, change for steps $(d=0.42$, $95 \% \mathrm{CI}[-0.28,1.12], Z=1.17, p=.243)$ and effectiveness $(d=-.04,95 \% \mathrm{CI}[-0.46,0.38], Z=$ 
$-.18, p=.858$ ) and neither analysis showed evidence of significant heterogeneity (largest $\tau^{2}=$ .16 , largest $I^{2}=62.55 \%$, largest $\mathrm{Q}=2.67$, smallest $\left.\mathrm{p}=.102\right)$. Leahy et al.'s (2017) MeST group (Steps: $d=0.07,95 \%$ CI $[-0.84,0.20]$; Effectiveness: $d=-.04,95 \%$ CI $[-0.55,0.64]$ ) showed only trivial change in both indices. Their Life Review variant of MeST, however, showed moderate improvement in steps taken $(d=0.78,95 \%$ CI $[0.16,1.40])$ but not effectiveness $(d=-.12,95 \%$ CI $[-0.72,0.48])$.

In between-group comparisons at post-intervention assessment $(k=3) \mathrm{MeST}$ consistently outperformed control comparisons in steps taken $(d=0.82,95 \% \mathrm{CI}[0.48,1.17]$, $Z=4.66, p<.001)$ and effectiveness $(d=0.74,95 \% \mathrm{CI}[0.40,1.09], Z=4.25, p<.001)$ and neither analysis showed evidence of heterogeneity between studies $\left(\tau^{2}\right.$ and $I^{2}$ estimates both 0 , largest $\mathrm{Q}=.51$, smallest $\mathrm{p}=.775$ ). All studies showed evidence of outperforming control groups in steps and effectiveness (See Figure 6).

In between-group comparisons at follow-up assessment $(k=2)$ there were only smallto-moderate, but non-significant, differences between groups for steps $(d=0.49,95 \% \mathrm{CI}[-$ $0.23,1.21], Z=1.33, p=.185)$ but MeST groups outperformed controls in terms of effectiveness $(d=0.55,95 \% \mathrm{CI}[0.12,0.98], Z=2.51, p<.01)$. Leahy et al.'s (2017) MeST group outperformed controls in terms of steps taken $(d=0.86,95 \%$ CI $[0.24,1.49])$ but there was only a small-to-moderate and non-significant difference between groups for effectiveness $(d=0.45,95 \%$ CI $[-0.15,1.06])$. Their Life Review group showed the reverse pattern with only a trivial difference between groups for steps taken $(d=0.13,95 \%$ CI $[-0.46$, $0.72])$ and a small-to-moderate benefit, compared to controls, for effectiveness $(d=0.64$, $95 \%$ CI $[0.04,1.25])$.

There was mixed evidence for improvement in problem solving performance but MeST outperformed control groups at post-intervention in the three studies to assess this outcome. However, in the two studies to assess this outcome at follow-up, this benefit was 
lost by that assessment point.

Hopelessness

Three studies (Serrano et al., 2004; Raes et al., 2009; Celano et al., 2016) measured change in hopelessness using the Beck Hopelessness Scale (BHS). Pooled effect sizes across these studies suggested that MeST led to significant improvement in hopelessness from pre- to post- intervention $(d=0.94,95 \% \mathrm{CI}[0.57,1.31], Z=4.95, p<.001)$ and there was no evidence of heterogeneity between individual study effect sizes $\left(\tau^{2}=0.0, I^{2}=0.0 \%, Q(2)=\right.$ $1.17, p=.557)$. Serrano et al. $(2004 ; d=1.14 ; 95 \% \mathrm{CI}[0.48,1.81])$ and Celano et al. $(2016 ; d$ $=0.95 ; 95 \% \mathrm{CI}[0.44,1.47])$ both showed large effects whereas Raes et al., $(2009 ; d=0.53$; $95 \% \mathrm{CI}[-0.36,1.42])$ showed a smaller effect and the confidence intervals for this effect overlapped with zero. In the only assessment of change in hopelessness from postintervention to follow-up, Celano et al. (2016) showed little evidence of continued improvement after MeST $(d=-.25,95 \%$ CI[-0.76, 0.26]).

The pooled effect size for the two studies that compared MeST to a control condition (Celano et al., 2016; Serrano et al., 2004) suggested that MeST outperformed control comparisons $(d=-0.79,95 \% \mathrm{CI}[-1.43,-0.16], Z=-2.44, p=.015)$ and there was only small and non-significant heterogeneity between the studies $\left(\tau^{2}=.13, I^{2}=58.91 \%, Q(1)=2.43, p=\right.$ .119). Serrano et al. (2004) showed strong effects of MeST compared with their waitlist control $(d=-1.15,95 \%$ CI $[-1.80,-0.51])$ but the confidence intervals for Celano et al.'s (2016) comparison with their Positive Psychology control overlapped partially with zero $(d=$ $-.50,95 \%$ CI $[-1.00,0.01])$. By follow-up, Celano et al.'s MeST group showed only small but non-significant difference compared to their control group $(d=-.32,95 \%$ CI $[-0.84,0.20])$.

MeST lead to improvement in hopelessness by post-intervention assessment, at which point MeST marginally outperformed controls in active and passive controls. However, in the only study to examine the effects of MeST on hopelessness at follow-up this benefit 
compared to controls was lost.

Future episodic thinking

In the only study to examine improvement in the specificity of future episodic thinking through MeST (Blairy et al., 2008) there was evidence of substantial improvement from preto post- intervention $(d=-1.25,95 \% \mathrm{CI}[-2.04,-0.47])$ and MeST participants showed a large difference in future thinking compared to controls who received a psychoeducational intervention $(d=0.90,95 \% \mathrm{CI}[0.10,1.69])$. Ten MeST participants in this study also completed a follow-up assessment 12 weeks later but there was no evidence of continued improvement $(d=0.66,95 \% \mathrm{CI}[-0.17,1.48])$

\section{Discussion}

The present review considered the breadth of available literature regarding the effects of memory specificity training on problems with autobiographical memory specificity and the symptoms of emotional disorders and other associated processes. In our meta-analysis, MeST was associated with substantial improvement in memory specificity and depressive symptoms and outperformed passive control groups, and to a lesser extent active control groups, at post-intervention assessment. However, across most dependent variables MeST did not continue to outperform controls groups by follow-up assessment. MeST was also associated with improvements in problem solving performance and hopelessness relative to control groups at post-intervention. However, again, any benefit of MeST over controls was lost by follow-up assessment. MeST was associated with improvement in verbal fluency and episodic future thinking relative to control interventions at post-intervention. In the only study to examine verbal fluency at follow-up this group difference increased in size by follow-up assessment. Other indices of executive functioning such as digit span showed no significant improvement. There was mixed evidence that MeST influenced PTS symptoms or ruminative tendencies. 
It is of note that the effects of MeST seem to be confined to immediate postintervention assessment with little evidence of continued improvement, relative to controls, by follow-up assessment. The findings from this time point are to some extent skewed by Maxwell et al. (2015) and both of Leahy et al.'s (2017) groups whose participants were already highly specific at pre-intervention. Approximately $70 \%$ of memories recalled by participants in these studies at pre-intervention were specific and so they may already have been near to the ceiling for specificity before the intervention began. This level exceeds the mean post-intervention specificity scores found in studies that have used rAMS as an inclusion criteria: ranging from 59.5\% (Takano, Moriya, et al., 2017) to 66.7\% (WernerSeidler et al., 2018). It is perhaps not surprising that participants who had specificity levels above these levels showed little or no improvement in AMS or other associated symptoms or processes either. This represents a broader point for MeST trials, that participants are typically recruited because of their diagnostic status rather than because they show reduced specificity. MeST should only be offered to participants for whom rAMS is manifest (Takano, Moriya, et al., 2017; Werner-Seidler et al., 2018).

Also, five of the seven studies that included follow-up assessments and a control group, used 12-week follow-up durations. As such we were unable to examine whether the length of follow-up duration predicted differences in follow-up effect sizes.

It is also possible that post-interventions effects, particularly on memory specificity, represent mere practice effects and that the act of recalling specific memories in response to cue words improves specificity and its associated outcomes irrespective of the feedback and suggestions that are given within MeST. Future trials might utilise a wait-list control but where an AMT is administered every week, without feedback. Such a control would account for these practice effects, and if participants in the intervention group also complete AMTs in every session, this would also enable analyses regarding the potential mediational effects of 
the rate of change in specificity across treatment.

It is also possible that MeST did not outperform control groups at follow-up due to inadequate statistical power in the included studies. The majority of MeST trials appear to base their sample size estimates on the effect sizes for change in memory specificity across time and not on the effects of MeST on the symptoms of emotional disorder across time or the extent to which MeST changes symptoms relative to an active control condition. The average sample size in MeST groups in the studies reviewed here is approximately 20. Among the clinical trials which contrast the effects of MeST with a control group, these effects are for the most part small to moderate. This suggests that a $N$ of approximately 120 $(n=60)$ would be necessary in order to detect effects of this size on disorder symptoms in mixed measures designs with two groups and at two time points where alpha is .05 and with $90 \%$ power. No MeST study to-date has a sample of this size. However, it must be noted that the development of MeST has followed a systematic process from basic experimental science to clinical translation, beginning with uncontrolled pilot investigations in small samples and, more recently, comparisons with active control groups in larger samples. This process is in accordance with guidelines regarding the stages of intervention development (Rounsaville, Carroll, \& Onken, 2006). Although replication with larger samples is warranted, such studies are already inevitable given MeST's ongoing progression from basic science to clinical translation and the accumulation of evidence in support of MeST's effects on rAMS.

Compared with investigations of depressive symptoms, relatively fewer studies examined the processes associated with rAMS and emotional disorders in their MeST trials, and fewer still investigated the same processes. Nevertheless, there is consistent evidence that MeST leads to improvement in problem solving skills and hopelessness and that this persists into follow-up assessment. There is also some evidence that it leads to improvement in episodic future thinking and verbal fluency. More studies must explore the effects of MeST 
on these process outcomes before more robust conclusions can be drawn. In particular, more studies must test the hypothesis that improvements in these processes in turn leads to improvements in the symptoms of emotional disorders. One process variable that is particularly neglected is the extent to which MeST improves episodic future thinking. This variable has elsewhere been associated with reduced specificity and emotional disorders (D’Argembeau, Raffard, \& Van der Linden, 2008; Hallford, Austin, Takano, \& Raes, 2018) and it plays an important role in how individuals define themselves across time and plan for their future (Danion et al., 2005; de Oliveira, Cuervo-Lombard, Salamé, \& Danion, 2009). The one MeST study to examine episodic future thinking (Blairy et al., 2008) also included some elements of future thinking training in their MeST protocol. It would be interesting to examine whether MeST alone can influence future thinking or whether it must be combined with future thinking training in order to elicit these effects.

Regarding the acceptability and feasibility of MeST, Werner-Seidler et al. (2018) found that drop-out was no greater than a psychoeducational and counselling control and that participants in both groups were equally likely to recommend the intervention to a friend. Importantly, compared to control participants, participants in the MeST group reported higher ratings of how logical their intervention seemed and they reported feeling more optimistic about the success of the intervention. In qualitative analyses of MeST, for some people, MeST has been reported as not seeming relevant to the day-to-day memory dysfunctions they experienced (Leahy et al., 2017) and yet other people have remarked that they were pleased that this often-neglected aspect of their symptoms was being focused on (Eigenhuis et al., 2017). To participants in one study, the cue words often seemed abstract and it was difficult to think of a memory with which they were associated (Leahy et al., 2017). In the studies sampled, of the 256 people that have participated in MeST trials, only 7 participants $(2.7 \%)$ have dropped out during the intervention (see Table 1). These findings suggest that MeST is 
acceptable and feasible to participants but more studies must examine these variables as MeST is applied in other settings and with other participant groups.

\section{Recommendations for the future}

\section{Mechanism of action}

Perhaps the most significant limitation for existing MeST trials is the lack or inadequacy of the controls against which MeST has been compared. The initial pilot trial lacked any control (Raes et al., 2009) and several other trials utilised a waitlist control (Moradi et al., 2014; Neshat-Doost et al., 2013; Takano, Moriya, et al., 2017). The remaining trials have used controls with therapist interaction where other mechanisms involved in emotional disorders are targeted such as in basic psychoeducation or conversation training (Blairy et al., 2008), psychoeducation with counselling (Werner-Seidler et al., 2018) social support training (Ricarte et al., 2012), cognitive processing training where trauma experiences are reprocessed (Maxwell et al., 2016) and a Positive Psychology intervention (Celano et al., 2016). One other study has utilised a workbook control without therapist interaction (Leahy et al., 2017). These controls enable researchers to draw more valid conclusions regarding the effects of MeST but notably few of these allow researchers to examine the actual mechanisms of action within MeST. Below we make several recommendations for future research that would enable such examinations.

Besides our recommendation that MeST trials might include a control group where participants complete the AMT without feedback, it is also possible that the effects of MeST are attributable to exposure to negative affect and perhaps also the activation of positive affect through recall of positive events. Amongst participants who recall specific events, it may be that the effects of MeST are strongest, or perhaps only exist amongst people who recall and re-experience sufficiently negative and positive events from their past. Future studies might utilise controls where there is exposure to the emotions associated with 
memories where no training on specificity is given.

In a recent review of the current state-of-the-art regarding depression and its treatment, Otte et al. (2016) remark that some of the effects of psychological interventions for depression might be attributable to non-specific therapeutic effects related to the therapist and the qualities of their relationship with the client. Although control conditions which utilise other therapist-delivered interventions are valid in this respect, one other way to control for such effects is to adapt interventions for delivery using computer-based or online media such that interaction with a therapist is not required such as in c-MeST. To date, c-MeST has been used amongst people who have shown rAMS within the AMT (Takano et al., 2017) and has yet to be used amongst clinical groups. Further testing of c-MeST would allow researchers to conclude whether the effects of MeST are attributable to non-specific therapist factors, and hence why responsiveness to MeST in terms of the symptoms of emotional disorders has been similar to other clinician-administered psychoeducation (Blairy et al., 2008) and counselling (Werner-Seidler et al., 2018).

Also, with the exception of remotely-delivered variants such as c-MeST, MeST has mostly been delivered in a group format. It remains unclear whether the effects of MeST are attributable to the mere interaction with other people or, whether its effects are attributable to the sharing of autobiographical memories with others or the observation of other people's memory retrieval process. There is a growing body of evidence regarding the social function of sharing autobiographical memories in terms of developing or consolidating closeness and intimacy with others (Alea \& Bluck, 2003, 2007; Beike, Brandon, \& Cole, 2016). It is possible that sharing memories within a group format leads to enhanced intimacy between the members of the group and it may be that the forming of bonds with others and the support that this confers is the mechanism of action in MeST. As such, future research could compare individual MeST to group MeST, or where individual face-to-face MeST may not be 
financially feasible, c-MeST might be compared to typical, group MeST. In addition, future MeST trials might also assess closeness between group members to examine whether this mediates treatment effectiveness.

Similarly, it is unclear from the studies reviewed here, how much training is necessary in order to make MeST deliverers proficient in coding memories and offering feedback to participants whilst also coordinating group interactions. As such, it is also unclear to what extent studies differed in terms of the training they gave to the deliverer of MeST. Future research must quantify this important variable whilst also quantifying therapist adherence to the intervention protocol.

MeST protocols also differ in terms of the timing and order in which cue words of different valence are introduced. The original trial begins with positive and neutral cues and introduces negative cues at a later stage when it is assumed that participants are more able to deal with negative affect (Raes et al., 2009). Other studies include a new cue valence in each session or introduce negative cues earlier in treatment (Neshat-Doost et al., 2013). There is as yet no empirical basis for this ordering but, as the qualitative analyses conducted by some authors would suggest (Leahy et al., 2017; Maxwell et al., 2016), the introduction of different cue valences can have an important impact on participants' experiences of MeST. Specifically, some participants have reported that recalling memories following positive cue words can help them deal with the negative affect evoked by recalling memories from negative cue words (Maxwell et al., 2016) and yet other participants have reported that positive cue words can themselves evoke negative affect due to the retrieval of memories of depressing thoughts (e.g., when cued by happy a participant might think 'I'll never be happy again'; Eigenhuis et al., 2017). Future studies might investigate whether introducing cues of different valences in different orders between sessions influences treatment effectiveness as well as treatment adherence and drop-out. 


\section{Sample characteristics}

Many of the symptoms of depression, and in particular aspects of high negative affect or low positive affect, as well as mechanisms such as rAMS, are evident within other psychological disorders including bipolar disorder, eating disorders, and borderline personality disorder (Williams et al., 2007). It follows that MeST may hold promise amongst people with these disorders too. Two studies explored the effects of MeST on the symptoms of depression within people with schizophrenia (Blairy et al., 2008; Ricarte et al., 2012) but neither of these studies examined the effects of MeST on schizophrenia symptoms. Future research with other clinical groups should also report their effects on primary diagnoses and not just depressive symptoms. Another sample of participants that merits particular focus is groups of elderly people. rAMS may be particularly prominent amongst elderly people, and particularly those with depression (Wilson \& Gregory, 2018). Although several investigations included here already examined MeST, or Life Review, with older participants (Leahy et al., 2017; Serrano et al., 2004), there has yet to be an examination of MeST with elderly people who are depressed or who show rAMS.

There is a notable absence of any trials examining the effects of MeST on people at risk of disorder despite evidence that rAMS can predict a worsening of symptoms particularly in people exposed to trauma or in people in remission (Kleim \& Ehlers, 2008; Sumner et al., 2010). As Werner-Seidler et al. (2017) remark, one way to alleviate the significant burden placed on individuals and societies by emotional disorders is to prevent them from ever emerging. It is in this space that interventions such as MeST might offer particular promise. Such an approach could take two forms: either that MeST would be offered to people who have already been identified as having low specificity, or that it would be offered to those who show sub-clinical symptoms of depression. Regarding the former, Takano et al. (2017) have shown that is possible to offer MeST to people who do not have a clinical diagnosis but 
who might show rAMS, and in doing so, it is possible to improve their specificity significantly. That their c-MeST programme can be delivered to large cohorts of participants means that it offers particular promise in this respect as one might be able to use c-MeST in large samples of community participants and then examine change in symptoms over a year or more.

\section{Outcome assessment}

There are several ways in which future research might build upon existing MeST studies to further elucidate the effects of MeST. First, with one exception (Werner-Seidler et al., 2018) the remaining studies reviewed here explored change in self-reported symptoms across the intervention. Future research in this area might build on existing findings by using diagnostic criteria and examine the effects of MeST on caseness.

Only one of the studies reviewed here measured memory specificity using an alternative task to the AMT (Raes et al., 2009). Typically, within investigations of cognitive training programmes, a transfer task is utilised to examine the extent to which the training effects generalise across tasks assessing similar constructs. That MeST studies do not typically use such tasks is not necessarily as much of a problem as it might be for other forms of cognitive training programmes where the training protocol is a modified version of the assessment protocol. In those instances, a transfer task is necessary in order to establish that the training effects are not merely due to practice and repetition. The MeST protocol entails much more than the mere cued recall that features within the AMT, although the foundation of the programme is nonetheless still cued recall. Nevertheless, future MeST studies would benefit from the use of transfer tasks to further examine whether the training effects are generalizable to other forms of memory specificity measurement. In addition, one might examine the transfer of MeST's effects to other related memory processes such as narrative coherence (Vanderveren, Bijttebier, \& Hermans, 2017). 
It would be interesting to examine whether MeST is similarly effective at improving post-traumatic stress symptoms and depression symptoms. Such a comparison would not only allow us to draw conclusions about the transdiagnostic utility of MeST but would also enable us to draw conclusions regarding the contribution of AMS to post-traumatic stress symptoms and depression symptoms. That only two studies examined change in posttraumatic stress symptoms and thirteen examined change in depression symptoms precludes us from making such a comparison here. Future meta-analyses in this area could investigate this possibility.

\section{Risk of bias}

Finally, it is of note that only one study within the sampled investigations pre-registered their trial (Werner-Seidler et al., 2018). For the remaining studies it is unclear to what extent their reporting of their study aligns with their planning and conduct of the study, and as such a high risk of bias is possible. Most studies include a CONSORT diagram detailing the number of participants that were included in their study and how many of these were included in their analyses. However, for the majority of investigations it is unclear, given their reporting of their trial methodology, to what extent the intervention deliverers and participants were blind to the intervention they were offering/receiving or the outcomes that the interventions were being tested by. It is also largely unclear what system was used to randomise participants into their groups, and where this was the case whether experimenters and intervention deliverers alike were blind to the sequence that randomised participants into these groups. This is not to invalidate MeST research, but future studies in this area must follow more modern practices to ensure research transparency and reduce the risk of bias. In particular, more studies must now pre-register their methodology in advance and they must also fully report all aspects of their methodology so that a full and open risk of bias assessment can be conducted.

\section{Episodic Specificity Inductions}


Several studies identified in our initial literature search and the references within these studies, utilised an Episodic Specificity Induction (ESI) amongst healthy participants in order to explore the effects of specificity on other aspects of cognition. In these studies participants were asked to recall as much detail as possible from an experimentally contrived event presented to them on a video. Compared with control inductions where participants gave general impressions and opinions on the video or solved math problems following the video without questioning, the ESI was associated with enhanced problem solving abilities (Jing et al., 2016; Madore \& Schacter, 2014), enhanced creativity and divergent thinking (Madore, Jing, \& Schacter, 2016) and the generation of alternative positive outcomes to hypothetical negative scenarios (Jing, Madore, \& Schacter, 2017).

In line with the findings presented here, there is also evidence that the ESI can improve problem solving abilities amongst participants with depression (McFarland, Primosch, Maxson, \& Stewart, 2017). These studies suggest that when primed to think in a specific manner, people are better able to imagine possible future events and anticipate problems that might emerge in the future whilst thinking either about creative solutions to these problems or alternative outcomes to them. As has been discussed, future MeST investigations should include measures of episodic future thinking. It would be interesting to also examine whether MeST leads to improvements in the ability to generate alternative positive outcomes to negative events (Jing et al., 2017) given the suggestion that depression and other emotional disorders are characterised by the overestimation of negative outcomes (Macleod \& Byrne, 1996; Miloyan, Pachana, \& Suddendorf, 2014).

ESI research also suggests that inducing people to think in a specific way about episodic events can also enhance the internal or episodic detail with which past events are recalled (Madore, Gaesser, \& Schacter, 2014) and future events are imagined (Madore, Addis, \& Schacter, 2015). Research suggests that memory specificity and episodic detail are 
separable constructs and that they both predict different aspects of depressive symptomatology (Kyung, Yanes-Lukin, \& Roberts, 2016). The variant of MeST used within Blairy et al. (2008) included aspects of game-ificiation where participants were rewarded depending on the level of detail recalled during treatment, however, they did not examine whether MeST improved the amount of internal or episodic detail that participants retrieved. Future research should explore the effects of MeST on episodic detail.

Also, although it has been suggested that rAMS may be associated with deficits in emotion regulation (Williams et al., 2007), and ESI research supports this suggestion (Jing et al., 2016), there were no examinations of whether MeST improved this ability. Jing et al. (2016) assessed emotion regulation using a Cognitive Reappraisal task. In this task, participants are presented with several situations with negative outcomes. They are then asked to imagine how anxious they would feel in these situations, how likely they felt this negative outcome would be and how well they thought they would be able to cope with this outcome. Participants must then try to reinterpret the outcomes of these situations to make them more positive whilst reporting their thoughts, feelings and actions whilst doing so. Following the specificity induction participants reported the negative outcome seemed less likely, it provoked less anxiety and they felt they would be better able to cope with it if it occurred. Future research could examine whether similar effects are evident following MeST. The nature of the ESI highlights how, in MeST, it is not clear whether the details of situations being recalled are real or false or whether the situations being recalled by different participants are similarly valenced. Given the suggestion that participants with rAMS may possess this problem because of their tendency to avoid negative affect (Williams et al., 2007) it is possible that this avoidance continues in MeST. Researchers might adapt MeST such that it uses situations that are experienced within treatment and which the therapist can control, much like how the ESI uses hypothetical situations with which to induce specificity. 
Participants might be presented with hypothetical negative (e.g., failing an exam), positive (e.g., receiving a gift) and neutral (e.g., looking at a landscape) situations. After this, as in MeST, recall of sensory and contextual detail would be encouraged. By using such a design, therapists could control the affective value of the situations being recalled whilst also knowing for themselves what specific details must be recalled by the participant. As clinicians are able to probe memories in more detail whilst ensuring that participants are not avoiding negative emotional states, it is hoped that this would not only improve the effectiveness of MeST but also standardise it between participants.

\section{MeST and the treatment of emotional disorders}

A question remains as to where MeST might be positioned relative to other existing interventions and in routine clinical practices where other interventions are being practiced. No study has yet explicitly examined the utility of MeST as an adjunct to routine clinical practice, or what the costs of such integration might be, both financially and in terms of participant outcomes. As Craske (2018) suggests, there is a need for interventions which target specific mechanisms associated with disorder maintenance. This does not mean that MeST should be used in isolation, especially given that rAMS is unlikely to explain all of the variance in disorder symptoms that exists amongst clinical participants. Instead, we propose that MeST may be best used in tailored settings, amongst participants who exhibit rAMS, and in conjunction either with routine clinical practices where acute disorder symptoms are targeted or in conjunction with other interventions that target other disorder mechanisms, such as attention or interpretive biases.

\section{Conclusion}

In overview, MeST holds promise as a novel and targeted intervention for improving reduced autobiographical memory specificity (rAMS) and the symptoms of emotional disorder. However, our meta-analysis suggests that many of its effects are transitory, and do not persist 
into follow-up assessment. Future research is warranted. Strategies to enhance MeST's effects could be adopted and larger and more varied samples of participants could be employed, with control groups that allow for more robust examination of MeST's mechanism of action. Additional moderators and mediators of treatment must also be considered and MeST must be tested amongst at-risk samples with long-term follow-ups. 
Table 1.

\begin{tabular}{|c|c|c|c|c|c|c|c|c|}
\hline & & & Samp & e Characteri & tics & & & \\
\hline Diagnostic group & Age & Females & Dropout & $\begin{array}{l}\text { Baseline } \\
\text { Spec. }\end{array}$ & $\begin{array}{c}\text { Spec. } \\
\text { Measure }\end{array}$ & Comparison & Sessions & $\begin{array}{c}\text { FU } \\
\text { duration }\end{array}$ \\
\hline $\begin{array}{l}\text { Serrano et al., } 2004 \\
\text { Depression } \\
(N=43 ; n=20)\end{array}$ & $75.8(8.1)$ & $82.6 \%$ & 0 & $3.15(3.10)$ & Spec. total & Waitlist & 4 & \\
\hline $\begin{array}{l}\text { Blairy et al., } 2008 \\
\qquad \begin{array}{l}\text { Schizophrenia } \\
(N=27 ; n=15)\end{array}\end{array}$ & $41.2(11.4)$ & $46.7 \%$ & 0 & $4.99(2.30)$ & Spec. total & Psychoed. & 10 & 12 \\
\hline $\begin{array}{l}\text { Raes et al., } 2009 \\
\quad \text { Depression }(N=10)\end{array}$ & $47.4(7.6)$ & $100.0 \%$ & 2 & $.44(.28)$ & $\begin{array}{l}\text { Spec. prop. } \\
\text { of memories }\end{array}$ & MeST only & 4 & \\
\hline $\begin{array}{l}\text { Ricarte et al., } 2012 \\
\qquad \begin{array}{l}\text { Schizophrenia } \\
(N=50 ; \mathrm{n}=24)\end{array}\end{array}$ & $35.2(13.3)$ & $16.7 \%$ & 0 & $4.21(2.80)$ & Spec. total & $\begin{array}{l}\text { Social Skills } \\
\text { Training }\end{array}$ & 10 & \\
\hline $\begin{array}{l}\text { Neshat-Doost et al., } 2013 \\
\text { Depression } \\
(N=23 ; n=12)\end{array}$ & $14.9(1.9)$ & $46.8 \%$ & 0 & $.63(.15)$ & $\begin{array}{l}\text { Spec. prop. } \\
\text { of memories }\end{array}$ & Waitlist & 5 & 8 \\
\hline $\begin{array}{l}\text { Moradi et al., } 2014 \\
\qquad \text { PTSD }(\mathrm{N}=24 ; \mathrm{n}=12)\end{array}$ & $45.3(3.9)$ & $0.0 \%$ & 0 & $1.92(.90)$ & Spec. total & Waitlist & 4 & 12 \\
\hline $\begin{array}{l}\text { Maxwell et al., } 2016 \\
\qquad \operatorname{PTSD}(N=18 ; n=9)\end{array}$ & $23.9(5.5)$ & $81.0 \%$ & 0 & $7.75(2.32)$ & Spec. total & $\begin{array}{l}\text { Cognitive } \\
\text { Processing } \\
\text { Therapy }\end{array}$ & 6 & 12 \\
\hline $\begin{array}{l}\text { Celano et al., } 2016 \\
\text { Depression } \\
(N=65 ; n=33)\end{array}$ & $43.2(17.1)$ & $69.0 \%$ & 2 & & Not assessed & $\begin{array}{l}\text { Positive } \\
\text { Psychology }\end{array}$ & 6 & 6 \\
\hline
\end{tabular}




\begin{tabular}{|c|c|c|c|c|c|c|c|c|}
\hline \multicolumn{9}{|l|}{ Eigenhous et al. 2017} \\
\hline Depression $(N=32)$ & $41.7(9.8)$ & $57.7 \%$ & 0 & $.63(.28)$ & $\begin{array}{l}\text { Spec. prop. } \\
\text { of memories }\end{array}$ & MeST only & 5 & 12 \\
\hline \multicolumn{9}{|l|}{ Leahy et al., 2017} \\
\hline $\begin{array}{l}\text { Healthy }(N=66) \\
\operatorname{MeST}(n=22)\end{array}$ & $74.8(6.6)$ & $63.6 \%$ & 0 & $.71(.22)$ & $\begin{array}{l}\text { Spec. prop. } \\
\text { of cues }\end{array}$ & $\begin{array}{l}\text { Cognitive } \\
\text { Workbook }\end{array}$ & 4 & 12 \\
\hline Life Review $(n=22)$ & $74.1(3.7)$ & $77.3 \%$ & 1 & $.71(.16)$ & $\begin{array}{l}\text { Spec. prop. } \\
\text { of cues }\end{array}$ & $\begin{array}{l}\text { Cognitive } \\
\text { Workbook }\end{array}$ & 4 & 12 \\
\hline $\begin{array}{l}\text { Takano et al., } 2017 \\
\text { Healthy } \\
(\mathrm{N}=51 ; \mathrm{n}=29)\end{array}$ & $20.0(1.3)$ & $66.7 \%$ & 0 & $3.43(1.33)$ & Spec. total & Waitlist & 7 & 2 \\
\hline $\begin{array}{l}\text { Werner-Seidler et al., } 2018 \\
\text { Depression } \\
(N=59 ; n=31)\end{array}$ & $44.5(15.3)$ & $70.1 \%$ & 2 & $5.42(2.01)$ & Spec. total & $\begin{array}{l}\text { Psychoed. and } \\
\text { counselling }\end{array}$ & 5 & 12 \\
\hline
\end{tabular}

Notes. Sample characteristics of studies examining the effects of Memory Specificity Training (MeST) on autobiographical memory specificity and the symptoms of emotional disorders. Mean age (SD), the proportion of females, the number of participants who dropped out during the intervention and specificity scores from Autobiographical Memory Test (AMT; Williams \& Broadbent, 1986) at pre-intervention assessment (Baseline spec.) within the experimental group are also given. The way in which this specificity score was computed for each study is also given (Spec. total: total number of specific memories recalled; Spec. Prop of memories: total number of specific memories recalled divided by proportion of cues given minus number of omissions; Spec. prop. of cues; total number of specific memories recalled divided by proportion of cues given), as well as the nature of the control group (Psychoed.: Psychoeducation), if any was used, the number of MeST sessions and the length of follow-up (weeks). In the case of Leahy et al., 2017, scores are given for both the MeST group and also their Life Review group. 
Figure 1.

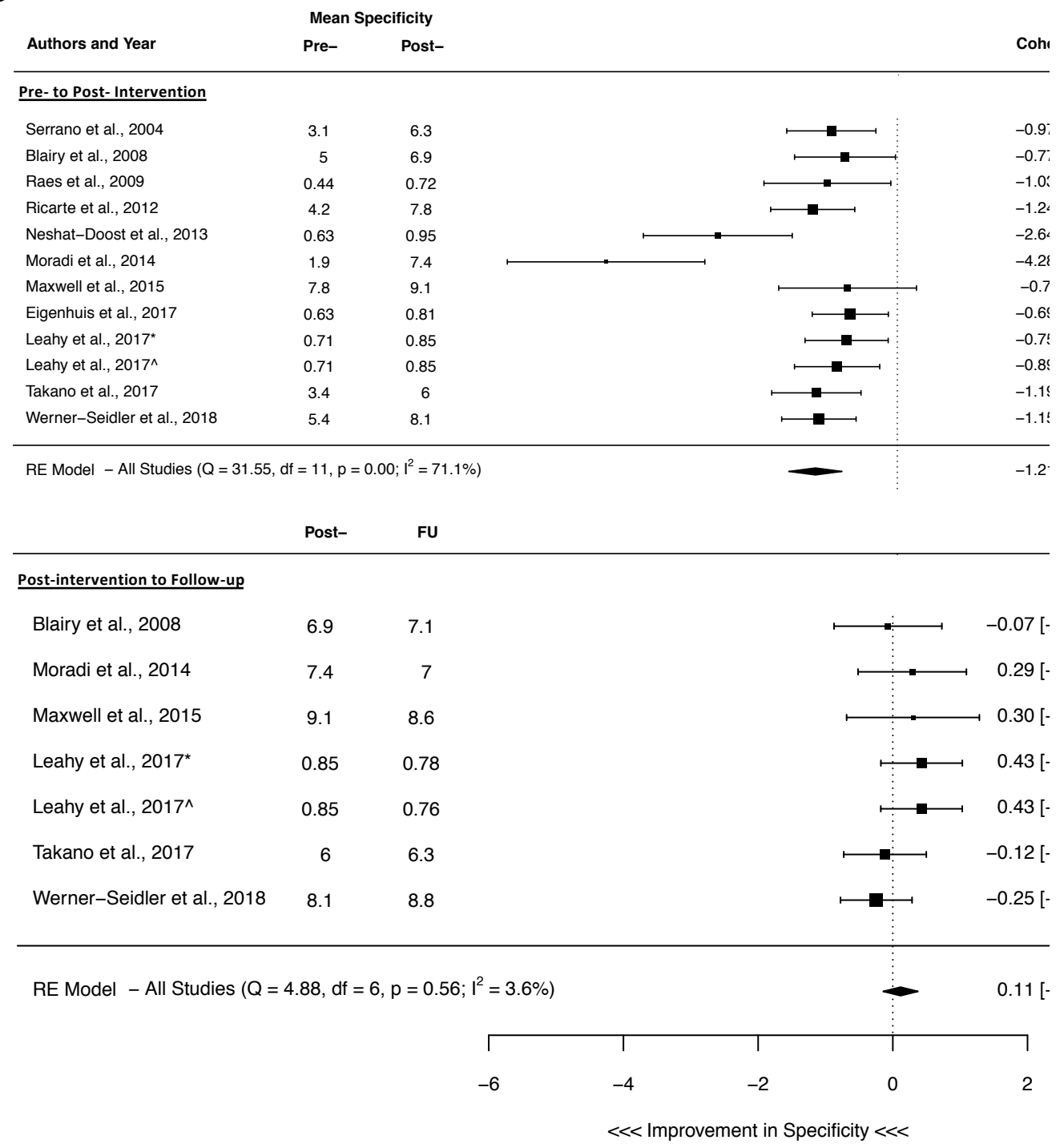

Note. Forest plot of effect sizes for change in specificity from pre- to post- interv from post-intervention to follow-up. 
Figure 2.

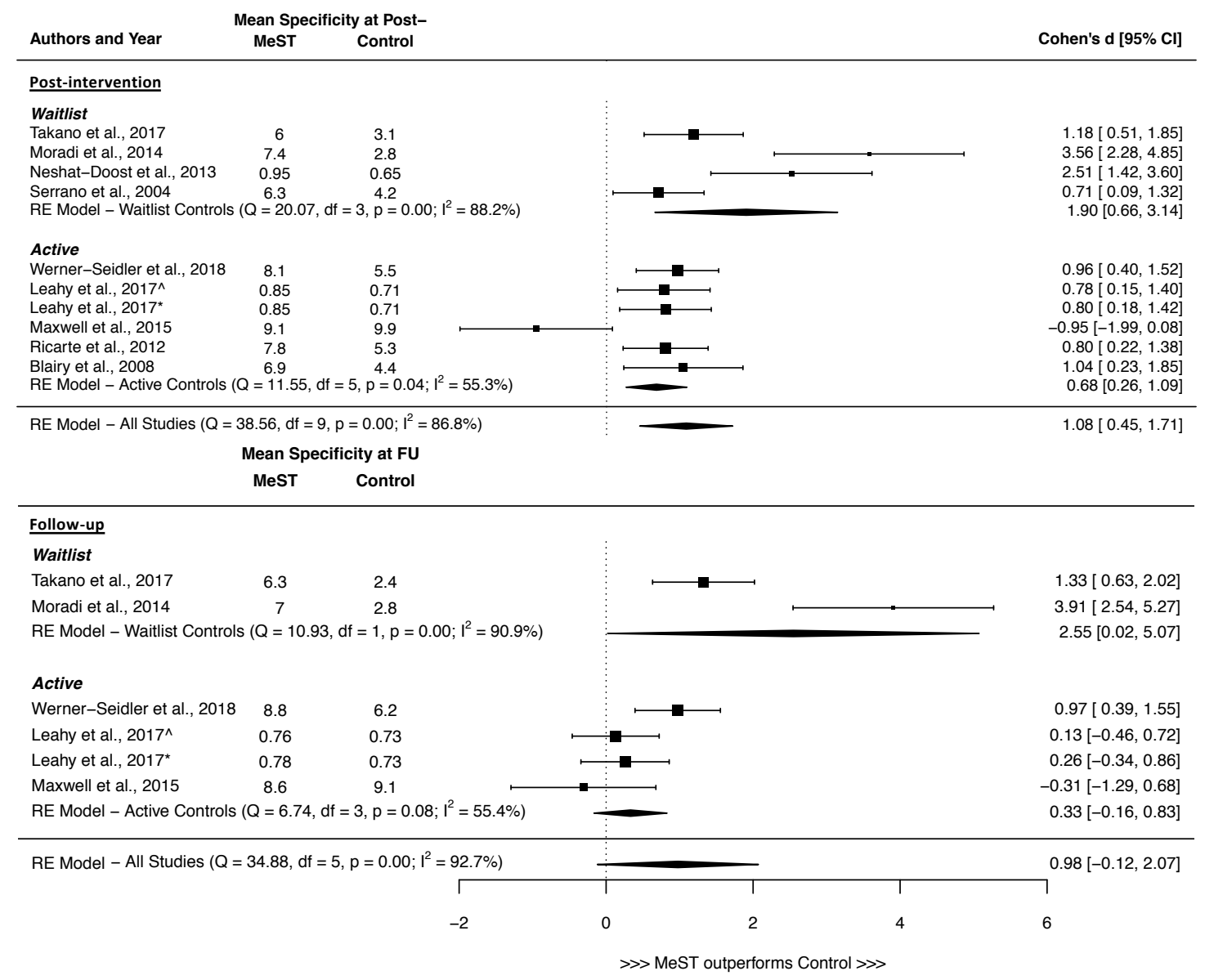

Note. Forest plot of effect sizes for group differences in specificity at post- intervention and from follow-up. 
Figure 3.

Authors and Year

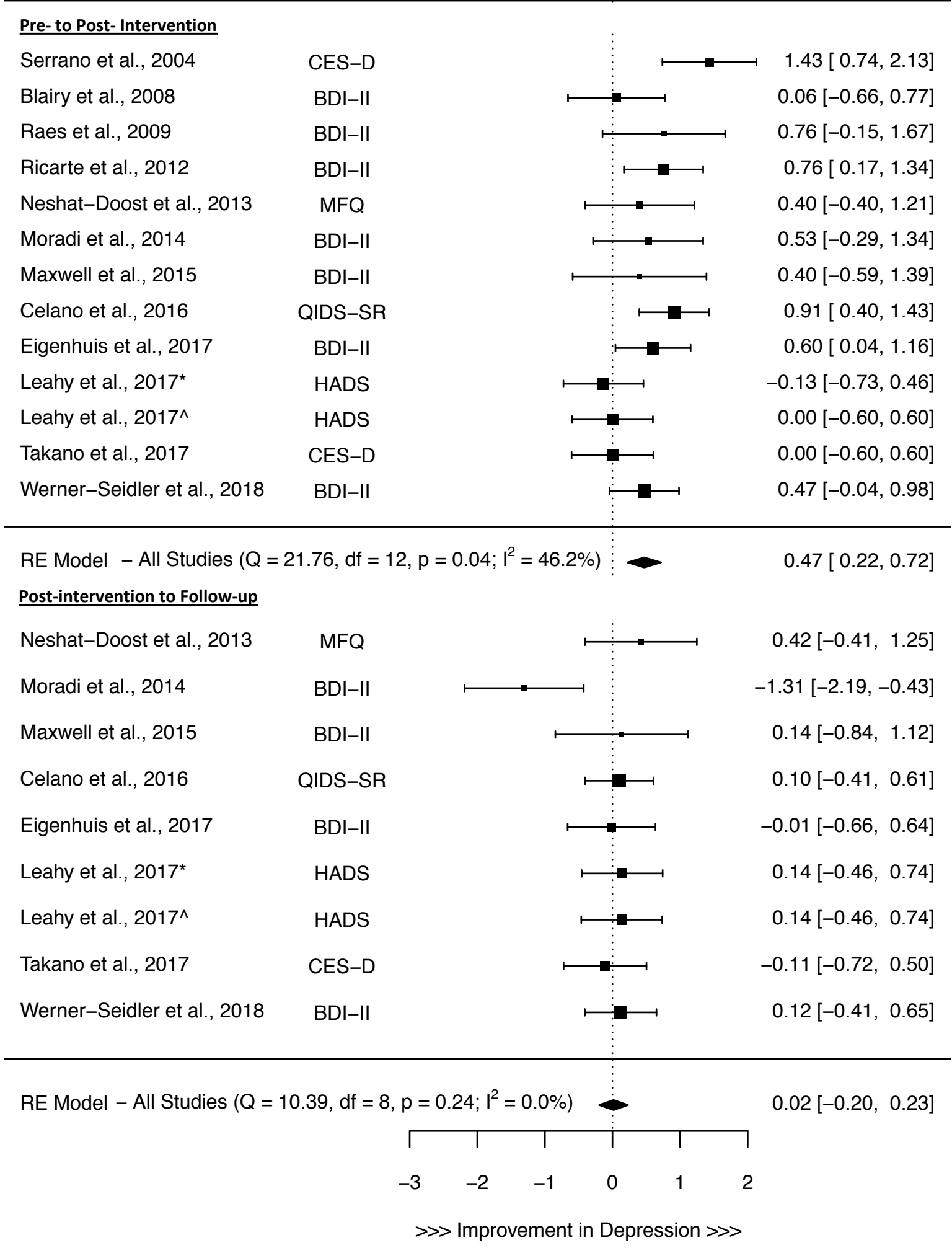

Note. Forest plot of effect sizes for change in depression symptoms from pre- to postintervention and from post-intervention to follow-up. Depression measures are given for each study (BDI-II; Beck Depression Inventory second version; HADS: Hamilton Anxiety and 
Depression Scale; QIDS-SR: Quick Inventory of Depressive Symptomatology, Self-Report; CES-D: Center for epidemiologic studies depression scale; MFQ: Mood and Feelings Questionnaire). 
Figure 4.

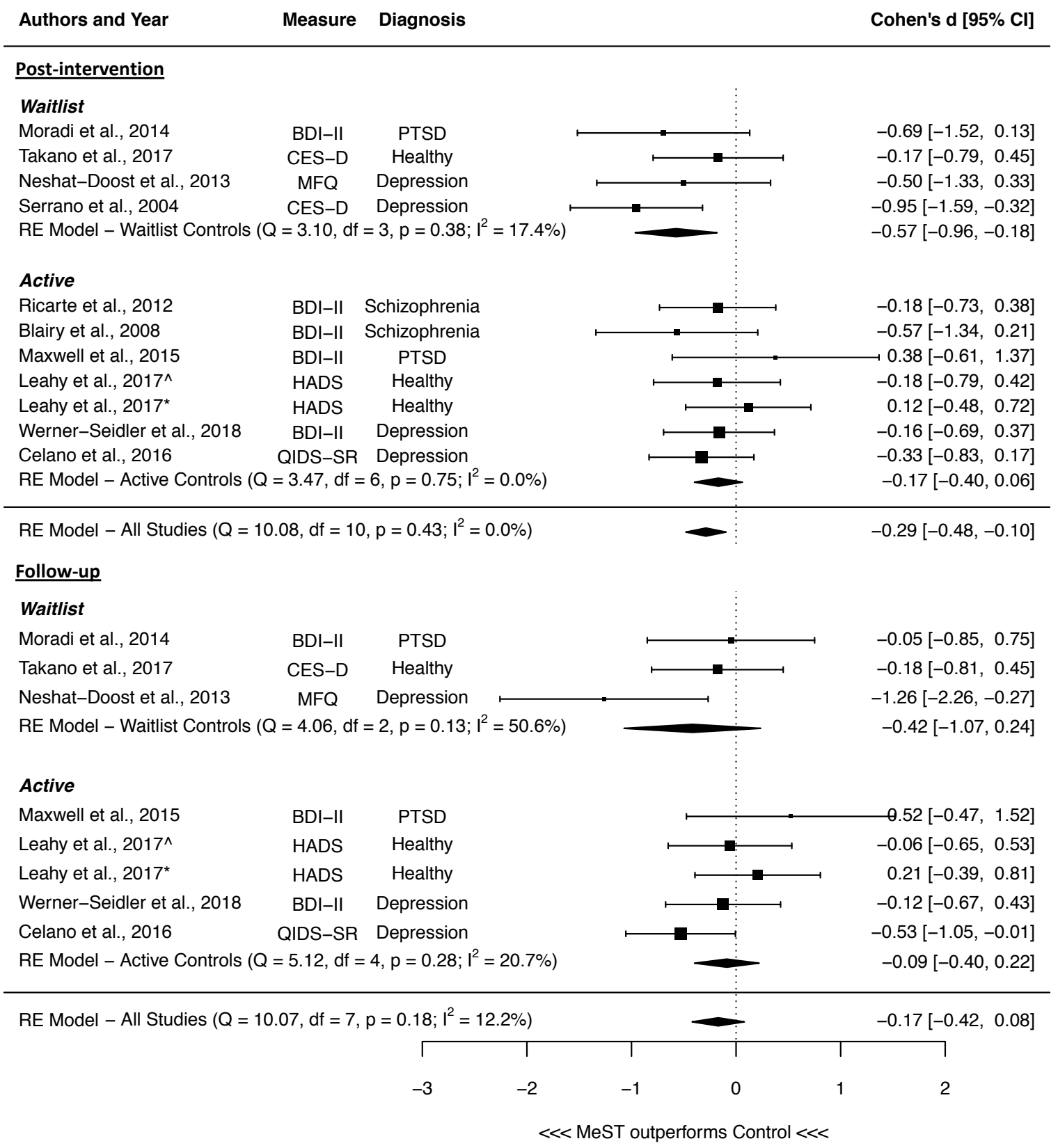

Note. Forest plot of effect sizes for group differences in depression at post- intervention and from follow-up. Diagnostic group (PTSD: Posttraumatic Stress Disorder) and depression measures are given for each study (BDI-II; Beck Depression Inventory second version; HADS: Hamilton Anxiety and Depression Scale; QIDS-SR: Quick Inventory of Depressive Symptomatology, Self-Report; CES-D: Center for epidemiologic studies depression scale; MFQ: Mood and Feelings Questionnaire). 
Figure 5.

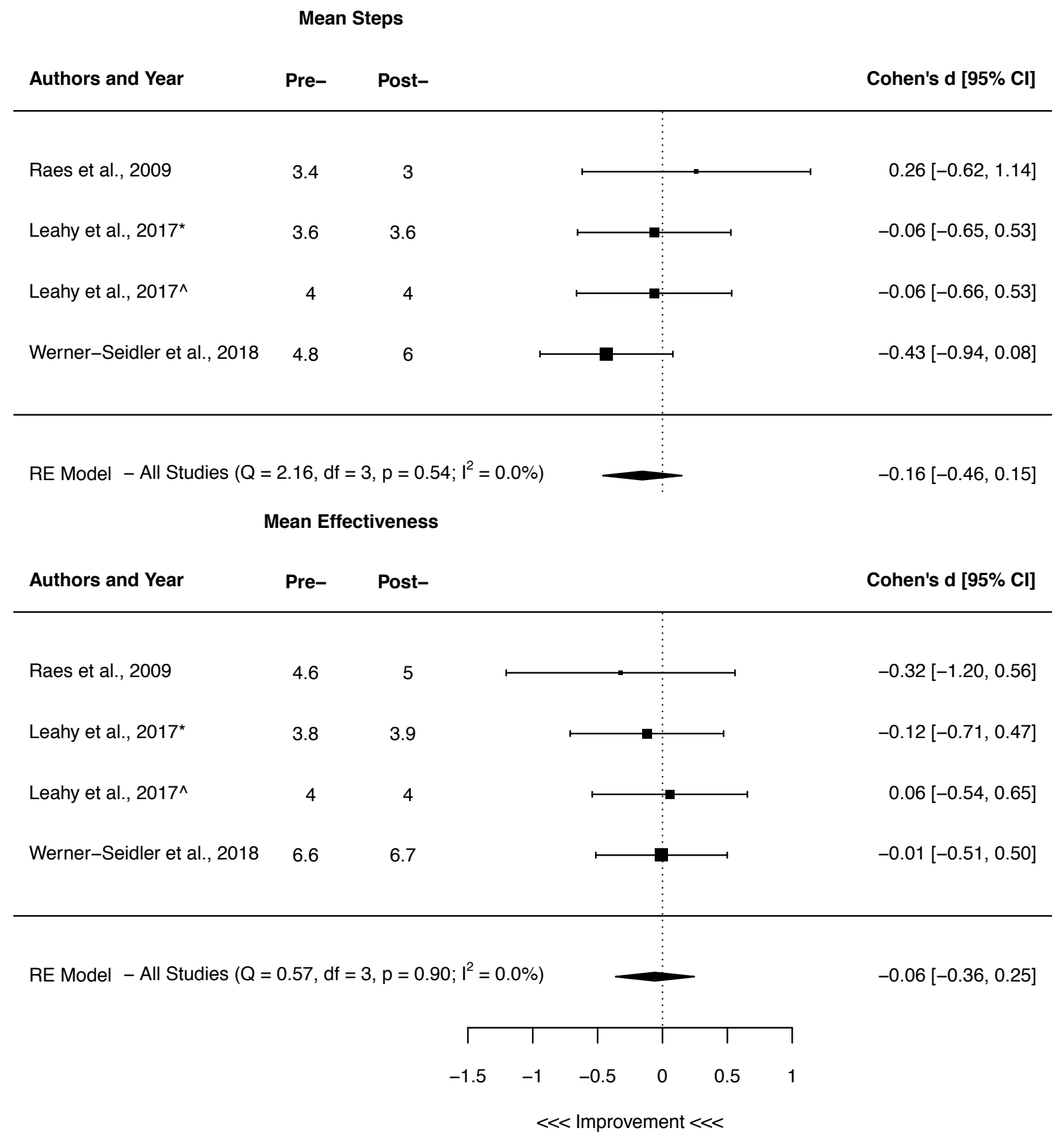

Note. Forest plot of effect sizes for change in performance in the Means-End Problem

Solving (MEPS) Task from pre- to post- intervention for steps-taken and effectiveness subscores. 
Figure 6.

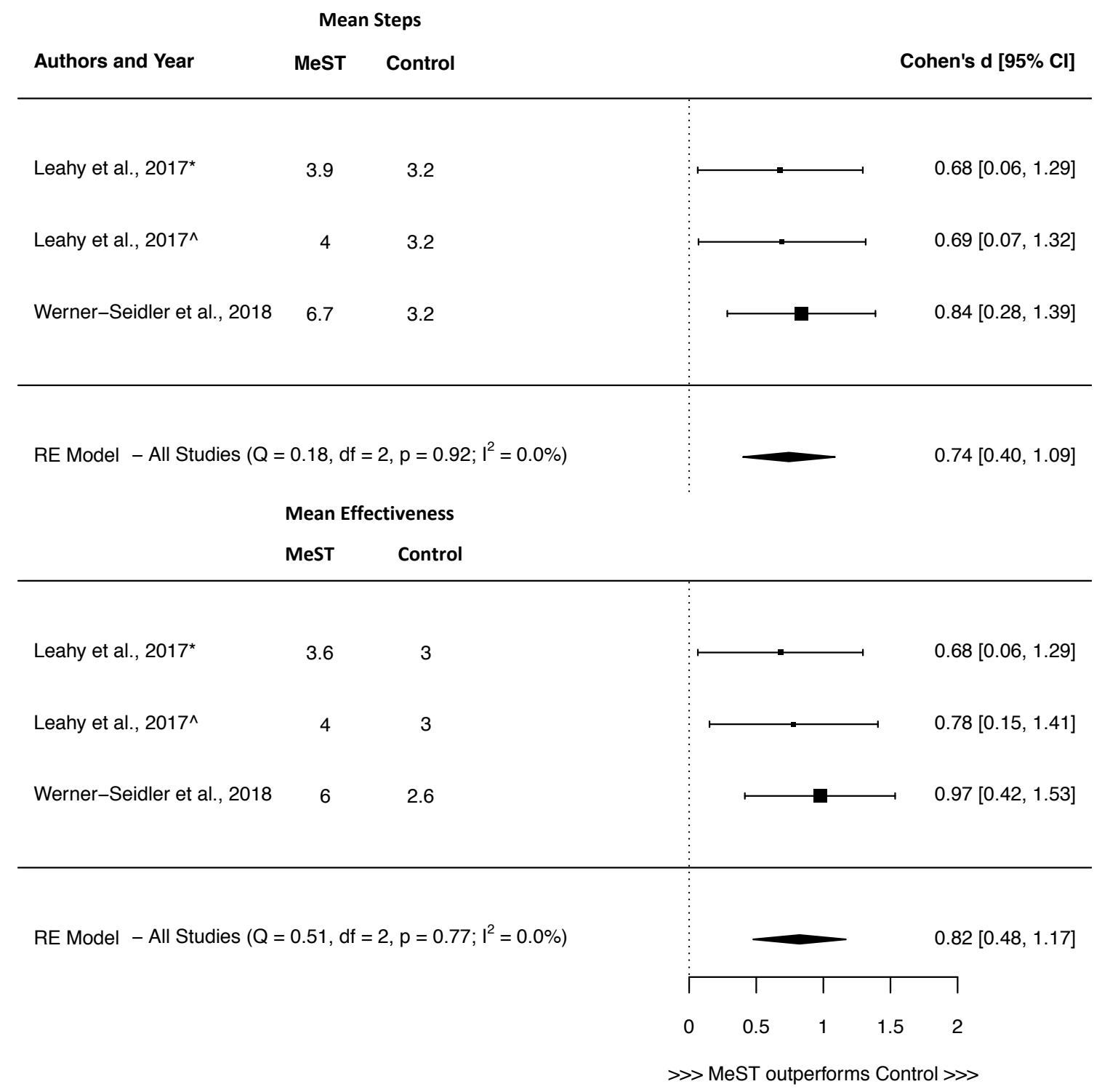

Note. Forest plot of effect sizes for between-group differences in performance in the MeansEnd Problem Solving (MEPS) Task at post- intervention for steps-taken and effectiveness sub-scores. 


\section{References}

Alea, N., \& Bluck, S. (2003). Why are you telling me that? A conceptual model of the social function of autobiographical memory. Memory, 11(2), 165-178. https://doi.org/10.1080/741938207

Alea, N., \& Bluck, S. (2007). I'll keep you in mind: The intimacy function of autobiographical memory. Applied Cognitive Psychology, 21(8), 1091-1111. https://doi.org/10.1002/acp.1316

Arie, M., Apter, A., Orbach, I., Yefet, Y., \& Zalzman, G. (2008). Autobiographical memory, interpersonal problem solving, and suicidal behavior in adolescent inpatients. Comprehensive Psychiatry, 49(1), 22-29. https://doi.org/10.1016/j.comppsych.2007.07.004

Beike, D. R., Brandon, N. R., \& Cole, H. E. (2016). Is sharing specific autobiographical memories a distinct form of self-disclosure? Journal of Experimental Psychology: General, 145(4), 434-450. https://doi.org/10.1037/xge0000143

Blairy, S., Neumann, A., Nutthals, F., Pierret, L., Collet, D., \& Philippot, P. (2008). Improvements in autobiographical memory in schizophrenia patients after a cognitive intervention: A preliminary study. Psychopathology, 41(6), 388-396. https://doi.org/10.1159/000155217

Celano, C. M., Beale, E. E., Mastromauro, C. A., Stewart, J. G., Millstein, R. A., Auerbach, R. P., ... Huffman, J. C. (2016). Psychological interventions to reduce suicidality in high-risk patients with major depression: a randomized controlled trial. Psychological Medicine, 47, 810-812. https://doi.org/10.1017/S0033291716002798

Conway, M. A., \& Pleydell-Pearce, C. W. (2000). The construction of autobiographical memories in the self-memory system. Psychological Review, 107(2), 261-288. https://doi.org/10.1037/0033-295X.107.2.261 
Craske, M. G. (2018). ScienceDirect Honoring the Past, Envisioning the Future : ABCT ' s 50th Anniversary Presidential Address. Behavior Therapy, 49(2), 151-164. https://doi.org/10.1016/j.beth.2017.05.003

Cuijpers, P., Cristea, I. A., Karyotaki, E., Reijnders, M., \& Huibers, M. J. H. (2016). How effective are cognitive behavior therapies for major depression and anxiety disorders? A meta-analytic update of the evidence. World Psychiatry, 15(3), 245-258. https://doi.org/10.1002/wps.20346

D’Argembeau, A., Raffard, S., \& Van der Linden, M. (2008). Remembering the Past and Imagining the Future in Schizophrenia. Journal of Abnormal Psychology, 117(1), 247251. https://doi.org/10.1037/0021-843X.117.1.247

Danion, J. M., Cuervo, C., Piolino, P., Huron, C., Riutort, M., Peretti, C. S., \& Eustache, F. (2005). Conscious recollection in autobiographical memory: An investigation in schizophrenia. Consciousness and Cognition, 14(3), 535-547. https://doi.org/10.1016/j.concog.2005.01.005

de Oliveira, H., Cuervo-Lombard, C., Salamé, P., \& Danion, J. M. (2009). Autonoetic awareness associated with the projection of the self into the future: An investigation in schizophrenia. Psychiatry Research, 169(1), 86-87.

https://doi.org/10.1016/j.psychres.2008.07.003

Eigenhuis, E., Seldenrijk, A., van Schaik, A., Raes, F., \& van Oppen, P. (2017). Feasibility and Effectiveness of Memory Specificity Training in Depressed Outpatients: A Pilot Study. Clinical Psychology and Psychotherapy, 24(1), 269-277. https://doi.org/10.1002/cpp.1995

Emsaki, G., NeshatDoost, H. T., Tavakoli, M., \& Barekatain, M. (2017). Treino específico de memória pode melhorar memória de trabalho e prospectiva em pacientes com comprometimento cognitive leve amnéstico. Dementia e Neuropsychologia, 11(3), 255- 
261. https://doi.org/10.1590/1980-57642016dn11-030007

Evans, J., Williams, J. M. G., O’loughlin, S., \& Howells, K. (1992). Autobiographical memory and problem-solving strategies of parasuicide patients. Psychological Medicine, 22(2), 399-405. https://doi.org/10.1017/S0033291700030348

Field, A. P., \& Gillett, R. (2016). How to do a meta-analysis. British Journal of Mathematical \& Statistical Psychology, 63(3), 665-694. https://doi.org/10.1348/000711010x502733

Hallford, D. J., Austin, D. W., Takano, K., \& Raes, F. (2018). Psychopathology and episodic future thinking: A systematic review and meta-analysis of specificity and episodic detail. Behaviour Research and Therapy, 102(September 2017), 42-51. https://doi.org/10.1016/j.brat.2018.01.003

Harris, L. S., Block, S. D., Ogle, C. M., Goodman, G. S., Augusti, E.-M., Larson, R. P., ... Urquiza, A. (2015). Coping style and memory specificity in adolescents and adults with histories of child sexual abuse. Memory, 8211(October), 1-13. https://doi.org/10.1080/09658211.2015.1068812

Hermans, D., Raes, F., \& Eelen, P. (2005). Mood and memory: A cognitive psychology perspective on maintenance of depressed mood and vulnerability for relapse. In J. Corveleyn, P. Luyten, \& S. J. Blatt (Eds.), The Theory and Treatment of Depression: Towards a Dynamic Interactionism Model (pp. 43-66). Leuven, Belgium/Mahwah, NJ: Leuven University Press/Lawrence Erlbaum Associates. https://doi.org/10.4324/9780203763995

Hitchcock, C., Werner-Seidler, A., Blackwell, S. E., \& Dalgleish, T. (2017). Autobiographical episodic memory-based training for the treatment of mood, anxiety and stress-related disorders: A systematic review and meta-analysis. Clinical Psychology Review, 52, 92-107. https://doi.org/10.1016/j.cpr.2016.12.003

Holmes, E., Blackwell, S. E., Burnett Heyes, S., Renner, F., \& Raes, F. (2016). Mental 
Imagery in Depression: Phenomenology, Potential Mechanisms, and Treatment Implications. Annual Review of Clinical Psychology, 12, 249-280.

https://doi.org/10.1146/annurev-clinpsy-021815-092925

Jing, H. G., Madore, K. P., \& Schacter, D. L. (2016). Worrying about the future: An episodic specificity induction impacts problem solving, reappraisal, and well-being. Journal of Experimental Psychology: General, 145(4), 402-418.

https://doi.org/10.1037/xge0000142

Jing, H. G., Madore, K. P., \& Schacter, D. L. (2017). Preparing for what might happen: An episodic specificity induction impacts the generation of alternative future events. Cognition, 169(September), 118-128. https://doi.org/10.1016/j.cognition.2017.08.010

Kleim, B., \& Ehlers, A. (2008). Reduced autobiographical memory specificity predicts depression and posttraumatic stress disorder after recent trauma. Journal of Consulting \& Clinical Psychology, 76(2), 231-242. https://doi.org/10.1037/0022-006X.76.2.231

Kyung, Y., Yanes-Lukin, P., \& Roberts, J. E. (2016). Specificity and detail in autobiographical memory: Same or different constructs? Memory, 24(2), 272-284. https://doi.org/10.1080/09658211.2014.1002411

Leahy, F., Ridout, N., Mushtaq, F., \& Holland, C. (2017). Improving specific autobiographical memory in older adults: impacts on mood, social problem solving, and functional limitations. Aging, Neuropsychology, and Cognition, 00(00), 1-29. https://doi.org/10.1080/13825585.2017.1365815

Linde, K., Rücker, G., Sigterman, K., Jamil, S., Meissner, K., Schneider, A., \& Kriston, L. (2015). Comparative effectiveness of psychological treatments for depressive disorders in primary care: network meta-analysis. BMC Family Practice, 16(1), 103. https://doi.org/10.1186/s12875-015-0314-X

Macleod, A. K., \& Byrne, A. (1996). Anxiety, Depression, and the Anticipation of Future 
Positive and Negative Experiences. Journal of Abnormal Psychology, 105(2), 286-289. https://doi.org/10.1037/0021-843X.105.2.286

Madore, K. P., Addis, D. R., \& Schacter, D. L. (2015). Creativity and memory: Effects of an episodic-specificity induction on divergent thinking. Psychological Science, 26(9), 1461-1468. https://doi.org/10.1177/0956797615591863

Madore, K. P., Gaesser, B., \& Schacter, D. L. (2014). Constructive episodic simulation: Dissociable effects of a specificity induction on remembering, imagining, and describing in young and older adults. Journal of Experimental Psychology. Learning, Memory, and Cognition, 40(3), 609-622. https://doi.org/10.1037/a0034885

Madore, K. P., Jing, H. G., \& Schacter, D. L. (2016). Divergent creative thinking in young and older adults: Extending the effects of an episodic specificity induction. Memory and Cognition, 44(6), 974-988. https://doi.org/10.3758/s13421-016-0605-z

Madore, K. P., \& Schacter, D. L. (2014). An Episodic Specificity Induction Enhances MeansEnd Problem Solving in Young and Older Adults. Psychology and Aging, 29(4), 913924.

Maxwell, K., Callahan, J. L., Holtz, P., Janis, B. M., Gerber, M. M., \& Connor, D. R. (2016). Comparative study of group treatments for posttraumatic stress disorder. Psychotherapy, 53(4), 433-445. https://doi.org/10.1037/pst0000032

McFarland, C. P., Primosch, M., Maxson, C. M., \& Stewart, B. T. (2017). Enhancing memory and imagination improves problem solving among individuals with depression. Memory and Cognition, 45(6), 932-939. https://doi.org/10.3758/s13421-017-0706-3

Miloyan, B., Pachana, N. A., \& Suddendorf, T. (2014). The future is here: A review of foresight systems in anxiety and depression. Cognition and Emotion. https://doi.org/10.1080/02699931.2013.863179

Moradi, A. R., Moshirpanahi, S., Parhon, H., Mirzaei, J., Dalgleish, T., \& Jobson, L. (2014). 
A pilot randomized controlled trial investigating the efficacy of MEmory Specificity Training in improving symptoms of posttraumatic stress disorder. Behaviour Research and Therapy, 56(1), 68-74. https://doi.org/10.1016/j.brat.2014.03.002

Neshat-Doost, H. T., Dalgleish, T., Yule, W., Kalantari, M., Ahmadi, S. J., Dyregrov, A., \& Jobson, L. (2013). Enhancing autobiographical memory specificity through cognitive training: An intervention for depression translated from basic science. Clinical Psychological Science, 1(1), 84-92. https://doi.org/10.1177/2167702612454613

Otte, C., Gold, S. M., Penninx, B. W., Pariante, C. M., Etkin, A., Fava, M., ... Schatzberg, A. F. (2016). Major depressive disorder. Nature Reviews Disease Primers, 2, 1-21. https://doi.org/10.1038/nrdp.2016.65

R Development Core Team, R. (2011). R: A Language and Environment for Statistical Computing. R Foundation for Statistical Computing. https://doi.org/10.1007/978-3-540$74686-7$

Raes, F., Williams, J. M. G., \& Hermans, D. (2008). Preliminary evaluation of a training program to increase autobiographical memory specificity in patients with depressive complaints. In 6th Special Interest Meeting on Autobiographical Memory and Psychopathology. Amsterdam, Netherlands.

Raes, F., Williams, J. M. G., \& Hermans, D. (2009). Reducing cognitive vulnerability to depression: A preliminary investigation of MEmory Specificity Training (MEST) in inpatients with depressive symptomatology. Journal of Behavior Therapy and Experimental Psychiatry, 40(1), 24-38. https://doi.org/10.1016/j.jbtep.2008.03.001

Ricarte, J. J., Hernández-Viadel, J. V., Latorre, J. M., \& Ros, L. (2012). Effects of eventspecific memory training on autobiographical memory retrieval and depressive symptoms in schizophrenic patients. Journal of Behavior Therapy and Experimental Psychiatry, 43, S12-S20. https://doi.org/10.1016/j.jbtep.2011.06.001 
Rounsaville, B. J., Carroll, K. M., \& Onken, L. S. (2006). A Stage Model of Behavioral Therapies Research: Getting Started and Moving on From Stage I. Clinical Psychology: Science and Practice, 8(2), 133-142. https://doi.org/10.1093/clipsy.8.2.133

Serrano, J. P., Latorre, J. M., Gatz, M., \& Montanes, J. (2004). Life review therapy using autobiographical retrieval practice for older adults with depressive symptomatology. Psychology and Aging, 19(2), 270-277. https://doi.org/10.1037/0882-7974.19.2.272

Sumner, J. A. (2012). The mechanisms underlying overgeneral autobiographical memory: An evaluative review of evidence for the CaR-FA-X model. Clinical Psychology Review, 32(1), 34-48. https://doi.org/10.1016/j.cpr.2011.10.003

Sumner, J. A., Griffith, J. W., \& Mineka, S. (2010). Overgeneral autobiographical memory as a predictor of the course of depression: A meta-analysis. Behaviour Research and Therapy, 48(7), 614-625. https://doi.org/10.1016/j.brat.2010.03.013

Takano, K., Gutenbrunner, C., Martens, K., Salmon, K., \& Raes, F. (2018). Computerized Scoring Algorithms for the Autobiographical Memory Test. Psychological Assessment, 30(2), 259-273. https://doi.org/10.1037/pas0000472

Takano, K., Moriya, J., \& Raes, F. (2017). Lost in distractors: Reduced Autobiographical Memory Specificity and dispersed activation spreading over distractors in working memory. Behaviour Research and Therapy, 94, 19-35. https://doi.org/10.1016/j.brat.2017.04.005

Takano, K., Ueno, M., Moriya, J., Mori, M., Nishiguchi, Y., \& Raes, F. (2017). Unraveling the linguistic nature of specific autobiographical memories using a computerized classification algorithm. Behavior Research Methods, 49(3), 835-852. https://doi.org/10.3758/s13428-016-0753-x

Van Vreeswijk, M. F., \& De Wilde, E. J. (2004). Autobiographical memory specificity, psychopathology, depressed mood and the use of the Autobiographical Memory Test: A 
meta-analysis. Behaviour Research and Therapy, 42(6), 731-743.

https://doi.org/10.1016/S0005-7967(03)00194-3

van Zoonen, K., Buntrock, C., Ebert, D. D., Smit, F., Reynolds, C. F., Beekman, A. T. F., \& Cuijpers, P. (2014). Preventing the onset of major depressive disorder: A meta-analytic review of psychological interventions. International Journal of Epidemiology, 43(2), 318-329. https://doi.org/10.1093/ije/dyt175

Vanderveren, E., Bijttebier, P., \& Hermans, D. (2017). The Importance of Memory Specificity and Memory Coherence for the Self: Linking Two Characteristics of Autobiographical Memory. Frontiers in Psychology, 8(December), 1-13. https://doi.org/10.3389/fpsyg.2017.02250

Viechtbauer, W. (2010). Conducting Meta-Analyses in R with the metafor Package. Journal of Statistical Software. https://doi.org/10.1103/PhysRevB.91.121108

Werner-Seidler, A., Hitchcock, C., Bevan, A., McKinnon, A., Gillard, J., Dahm, T., ... Dalgleish, T. (2018). A cluster randomized controlled platform trial comparing group MEmory specificity training (MEST) to group psychoeducation and supportive counselling (PSC) in the treatment of recurrent depression. Behaviour Research and Therapy, 105(February), 1-9. https://doi.org/10.1016/j.brat.2018.03.004

Werner-Seidler, A., Perry, Y., Calear, A. L., Newby, J. M., \& Christensen, H. (2017). School-based depression and anxiety prevention programs for young people: A systematic review and meta-analysis. Clinical Psychology Review, 51, 30-47. https://doi.org/10.1016/j.cpr.2016.10.005

Williams, J. M. G. (2006). Capture and rumination, functional avoidance, and executive control (CaRFAX): Three processes that underlie overgeneral memory. Cognition and Emotion. https://doi.org/10.1080/02699930500450465

Williams, J. M. G., Barnhofer, T., Crane, C., Hermans, D., Raes, F., Watkins, E., \& 
Dalgleish, T. (2007). Autobiographical memory specificity and emotional disorder. Psychological Bulletin, 133(1), 122-148. https://doi.org/10.1037/0033-2909.133.1.122 Williams, J. M. G., \& Broadbent, K. (1986). Autobiographical memory in suicide attempters. Journal of Abnormal Psychology, 95, 144-149.

Wilson, F. C. L., \& Gregory, J. D. (2018). Overgeneral autobiographical memory and depression in older adults: a systematic review. Aging and Mental Health. Taylor \& Francis. https://doi.org/10.1080/13607863.2017.1326461 